\title{
Design and Implementation of Vision System Aid in Windscreen Assembly
}

\author{
Abhishek Shukla \\ Department of Embedded System \\ International Institute of Information \\ Technology-Pune
}

\author{
Rohan Dwivedi \\ Department of Embedded System \\ International Institute of Information \\ Technology-Pune
}

\begin{abstract}
The automotive industries are more specific in automation of the present systems and making them responsive according to the environment. A control circuit for a wind shield wiper motor is described in this paper which will automatically make the response to the presence of moisture droplets on the windshields and vary its speed of motion accordingly. Different kind of sensors, actuators and mechanical designs were tested and finally we came up with the model using pic microcontroller. The cyst of this project is that the rain sensor is used to detect the amount of the rain and give the signal to the controller. The ADC in the controller detects the sensor input and gives the signal to the driver circuit. The motor driver actuates the motor to run at high speed or low speed based on the amount of rain detected. Initialization of the sensor is done when the vehicle is started and generates the power supply needed for the optimum functioning of the sensor. The threshold voltage value for the sensor is defined for the no rain and when it's raining, and the voltage will always be low. If the voltage reaches the minimum threshold value, the wiper will do its job at the maximum speed.
\end{abstract}

\section{Keywords}

PIC Microcontroller, Motor, Sensors, Wiper.

\section{INTRODUCTION}

In recent days the whole concept of making cars has changed and the companies are shifting towards providing more and more comfort to their customers. The invention of wiper dates back from 1903 in which brushes were used to clear the moisture and dust in the windshield. In most of the cars, the windshield wiper is operated manually and the speed of the wiper blades should be controlled manually. The working of the wiper is based on the conversion of the wiper motors linear motion into linear back and forth motion.

Inside the motor/gear assembly is the electronic circuit which senses when the wiper are in their down position. One such system designed for vision aid in windshield assembly. A control circuit for a windshield wiper motor is developed in which the wiper motor is made automatically responsive to the presence of moisture droplets on the windshield causing the wiper blades to sweep back and forth at a rate dependent upon the level of precipitation encountered. In this project, design and working of Windshield wiper speed control will be discussed taking into account the recent challenges in the windshield assembly.
The problem definition is to design a prototype for a PIC microcontroller (PIC18f458) based vision system aid in windshield assembly which controls the windshield wiper speed based on the moisture droplets settling on the windshield. For this a comprehensive study of sensors, actuators and mechanical design was done. Development of hardware module and the software algorithm has been done.

The basic method used for designing the circuit is that a rain sensor will detect the amount of rain and give the signal to the controller.

The ADC in the controller detects the sensor input and gives the signal to the driver circuit. The motor driver actuates the motor to run at high speed or low speed based on the amount of rain detected. The microcontroller used for the design is PIC18f458.

Here the discussion has been confined towards automatic windshield wiper which has a lot of advantages over the basic technology that is used normally in today's world. The subsequent sections will describe the basic working of the windshield wiper where the whole technique of how a windshield wiper works has been described. After that in the third section the hardware and software to be used are described. The fourth section covers the implementation part followed by result and conclusion.

\section{BASIC THEORY}

In this section, the general working of a windshield viper is described and the problem statement is analyzed. The basic idea of working of motor is necessary to make it autonomous for working in presence of moisture or rain.

\subsection{Working of windshield viper}

The working of the wiper is based on the conversion of the wiper motors linear motion into linear back and forth movement of the wiper blades. The wiper combines two mechanical technologies to perform their tasks:

A combination of electric motor and worm gear reduction power to the wipers and a neat linkage converts the rotational output of the motor into the back and forth motion of the wiper. 


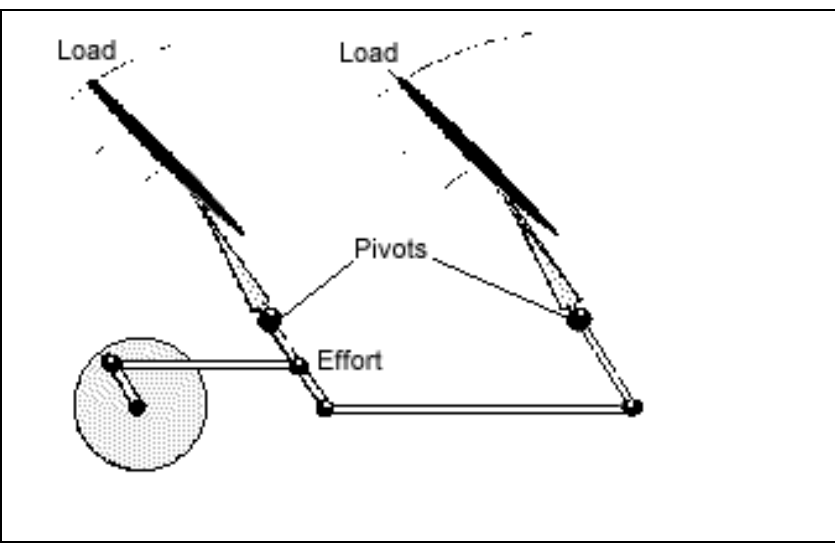

Figure1: Mechanics of wiper

Motor takes a lot of force to accelerate the wiper blades back and forth across the windshield so quickly. In order to generate this type of force, a worm gear is used on the output of a small electric motor. The worm gear reduction can multiply the torque of the motor by about 50 times and can slow it down with the same force. The output of the gear reduction operates a linkage that moves the wipers back and forth. Inside the motor/gear assembly is the electronic circuit which senses the wipers are in their down position. The circuit maintains power to the wipers until they are parked at bottom of the windshield, and then cuts the power to the motor. A short cam is attached to the output shaft of the gear reduction. This cam spins around as the wiper motor turns. The cam is connected to a long road; as the cam spins, it moves the rod back and forth. The long rod is connected to a short rod that actuates the wiper blade on the driver's side. Another long rod transmits the force from the driver's side to the passenger's side wiper blade.

\section{HARDWARE and SOFTWARE}

This section describes the hardware required for actual implementation and the software used for designing and simulating the test results. In the hardware section, practical implementation of the design using ADC and IR sensors are discussed. The speed of the viper is controlled electronically with the help of the microcontroller. In the software description, the programming flow is discussed using MATLAB and simulation software Proteus.

\subsection{Hardware Description}

The rain sensor is used to detect the amount of the rain and give the signal to the controller. The ADC in the controller detects the sensor input and gives the signal to the driver circuit. The motor driver actuates the motor to run at high speed or low speed based on the amount of the rain detected. The rain sensor will detect the moisture content on the windshield and based on the amount of moisture deposited on the windshield, the speed of the wiper is controlled. If the moisture or the rain dew deposited on the windshield is more, then the dispersion of the IR transmitter will be more. In that case, the system will turn on the wiper motor to activate at high speed using the driver circuit. If the moisture content is low, then the wiper motor is activated at low speed.
When the vehicle is started the rain sensor gets the supply power and it will scan for the moisture content in the windshield. The sensor is interfaced with the ADC of the controller. The ADC and the output port are initialized. Then the controller will start reading the sensor value. If there is no rain then the sensor output will be more than $4 \mathrm{~V}$ and if it is raining then the voltage will be less than $4 \mathrm{~V}$.When rain is detected then motor will be turned $\mathrm{ON}$ at slow speed. If the voltage drops less than $2.5 \mathrm{~V}$ then the motor will be turned $\mathrm{ON}$ to high speed.

The basic hardware requirements includes one PIC18f458 from microchip with program memory of $2 \mathrm{mb}$ and data memory of $4 \mathrm{~kb}$.The speed of this controller can vary up to 10 MIPS and its instruction set is 16 bit wide. It is having 8 channels ADC with PWM module and CAN bus.

The next component required is the rain sensor with a supply voltage of $10 \mathrm{~V}$ to $15 \mathrm{~V}$ and supply current less than $200 \mathrm{~mA}$. The temperature range of the sensor can be varied from $-20^{\circ} \mathrm{C}$ to $85^{\circ} \mathrm{C}$ with response time of 0.1 second.

The wiper motor used here is having power rating of $150 \mathrm{~W}$. The voltage required is $12-24 \mathrm{~V}$ and the current required is between $0.4-0.8$ amps. Torque generated by wiper motor is $5-25 \mathrm{Nm}$.

These are the basic building blocks of the hardware besides the resistors and capacitors that are required for any electronic circuit.

\subsection{Software Description}

The software used designing and simulating the test results were Proteus 7.0 and MatLab v8.14.05.

Proteus 7.0 is a Virtual System Modeling (VSM) that combines circuit simulation, animated components and microprocessor models to co-simulate the complete microcontroller based designs. This program allows users to interact with the design using on-screen indicators and/or LED and LCD displays and, if attached to the PC, switches and buttons. One of the main components of Proteus 7.0 is the Circuit Simulation -- a product that uses a SPICE3f5 analogue

Simulator kernel combined with an event-driven digital simulator that allows users to utilize any SPICE model by any manufacturer. Proteus VSM comes with extensive debugging features, including breakpoints, single stepping and variable display for a neat design prior to hardware prototyping.

MATLAB, which stands for MATrix LABoratory, is a state-ofthe-art mathematical software package, which is used extensively in both academia and industry. It is an interactive program for numerical computation and data visualization, which along with its programming capabilities provides a very useful tool for almost all areas of science and engineering. Unlike other mathematical packages, MATLAB cannot perform symbolic manipulations without the use of additional Toolboxes. Both 
these software's were used for doing all the simulation work and writing the program code. The algorithm used to develop the code is primarily implemented in the proteus and different test case results were obtained. Finally, the optimized algorithm was developed to run the application in real time.

\section{IMPLEMENTATION \& RESULT}

The implementation part deals with the real time environment. Power supply is given to the micro controller and the action is taken by the viper motor according to the program dumped on the micro controller.

\subsection{Hardware Implementation}

The basic control units of the hardware comprises of power supply unit, control switch, wiper motor, rain detector sensor ,motor driver circuit and the most important of all pic controller. Power supply unit maintains the continuous power to the controller and the wiper motor. Control switch is directly connected to the controller. Motor driver circuit is linked with the wiper motor and the controller. The command it gets from the controller is used to either drive the wiper motor or switch it off. Rain detection sensor detects the amount of moisture on the windscreen and accordingly sends the signal to the controller. a wiper motor control using a load sense resistor to monitor conditions confronting the wiper operation.

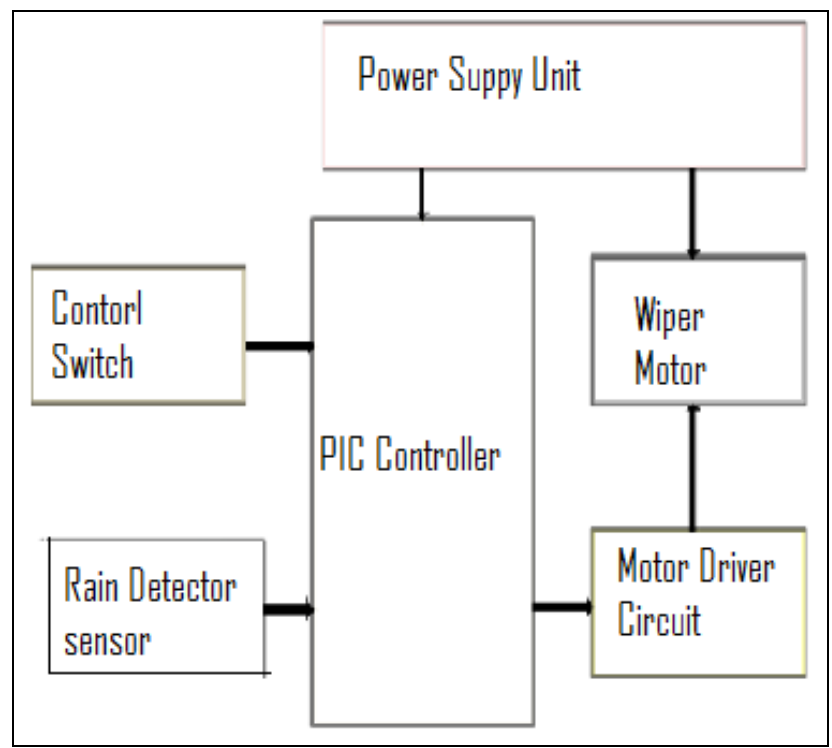

Figure2: Block Diagram of Hardware

The control switch can detect if the wiper is frozen to the window, moving too slowly, moving too quickly, etc. The power to the motor is then adjusted to achieve a desired speed, or, otherwise adjusted, based upon the detected conditions. Further, the direction of the motor may be reversed based upon the detected conditions. In a preferred embodiment, the load sense resistor is provided by a defroster filament strip. Alternatively, a temperature sensor may be used to detect the conditions confronting the wiper operation.

\subsection{Software Implementation}

The software part was implemented based on the following flow chart.

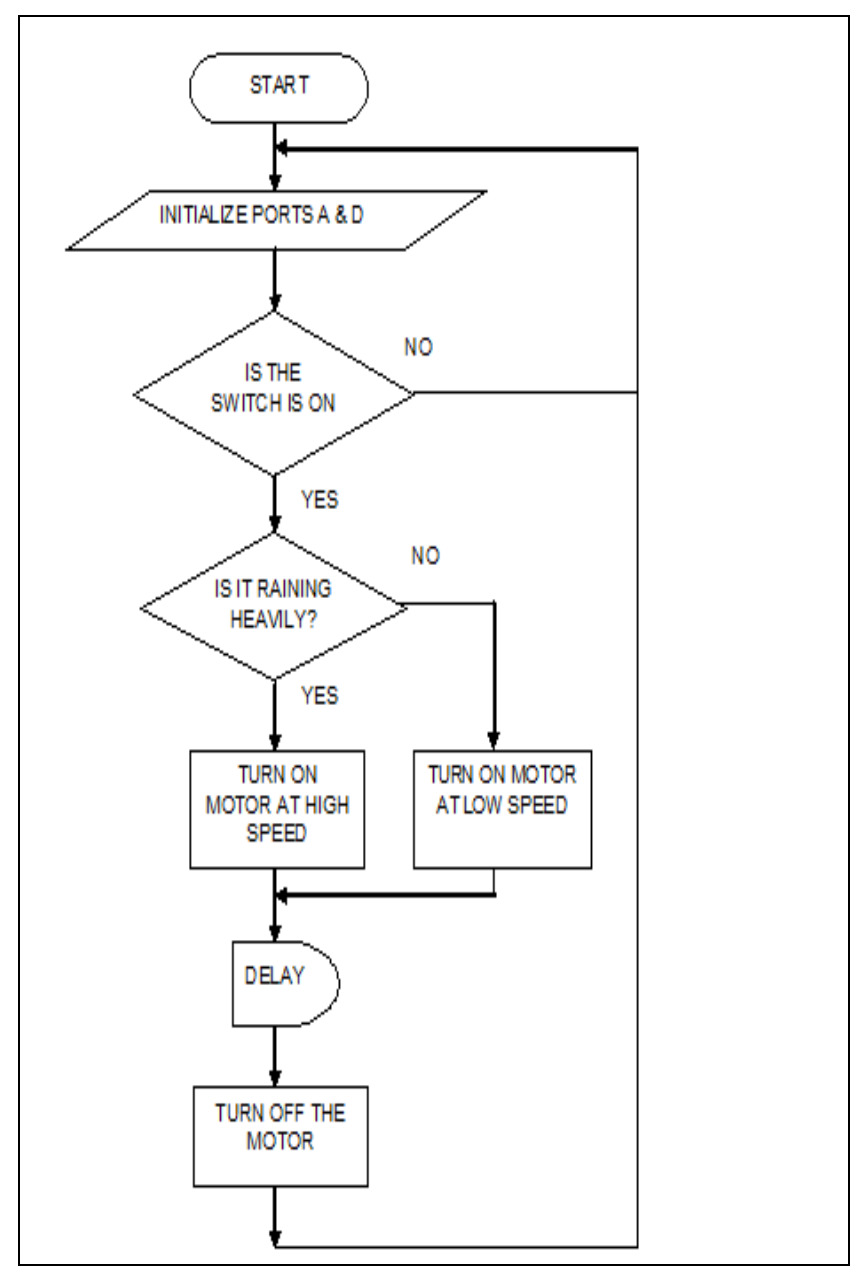

Figure3: Flow chart to develop the software

First initialization of ports $\mathrm{A}$ and $\mathrm{D}$ as input port and output port respectively of the ADC was done. Now switch is checked for it's ON or OFF status. If the switch is not ON the ports A and D are initialized again. And if the switch is $\mathrm{ON}$, condition of the rain is checked whether it's raining heavily or not. If yes, motor is turned on at the high speed and if the rain is not heavy, the motor speed is set to low. Delay is given after each stroke of the wiper blade and when there is no moisture left on the windscreen, motor is turned OFF.The delay sub routines are initialized before the main program is written. Vref $=\mathrm{Vdd}=5 \mathrm{v}$ has been set in the ADC. Now the analog-to-digital (A/D) Converter module has eight input channels for the PIC18F458 devices. This module has the $\mathrm{ADCON} 0$ and $\mathrm{ADCON} 1$ register definitions that are compatible with the PICmicro ${ }^{\circledR}$ mid-range A/D module. The $\mathrm{A} / \mathrm{D}$ allows conversion of an analog input signal to a corresponding 10-bit digital number. The A/D module has four registers. These registers are: $\mathrm{A} / \mathrm{D}$ result high resistor, $\mathrm{A} / \mathrm{D}$ result low resistor, A/D control resistor 0 and $\mathrm{A} / \mathrm{D}$ control resistor 1 . 
For doing the A/D conversion, A/D module was configured then the $\mathrm{A} / \mathrm{D}$ interrupt, after both has been done delay is set for the required acquisition time, then start the conversion and wait until the A/D conversion is finished and finally the $A / D$ result resistor is taken and then the necessary action.

\subsection{Result}

The IR sensor is connected to ADC channel O(AN0) of the controller. The LED's are connected to port D pin RDO and $\mathrm{RD} 1$. When the sensor output voltage is less than $3.5 \mathrm{~V}$, i.e. if the IR receiver detects only a partial IR ray from the IR

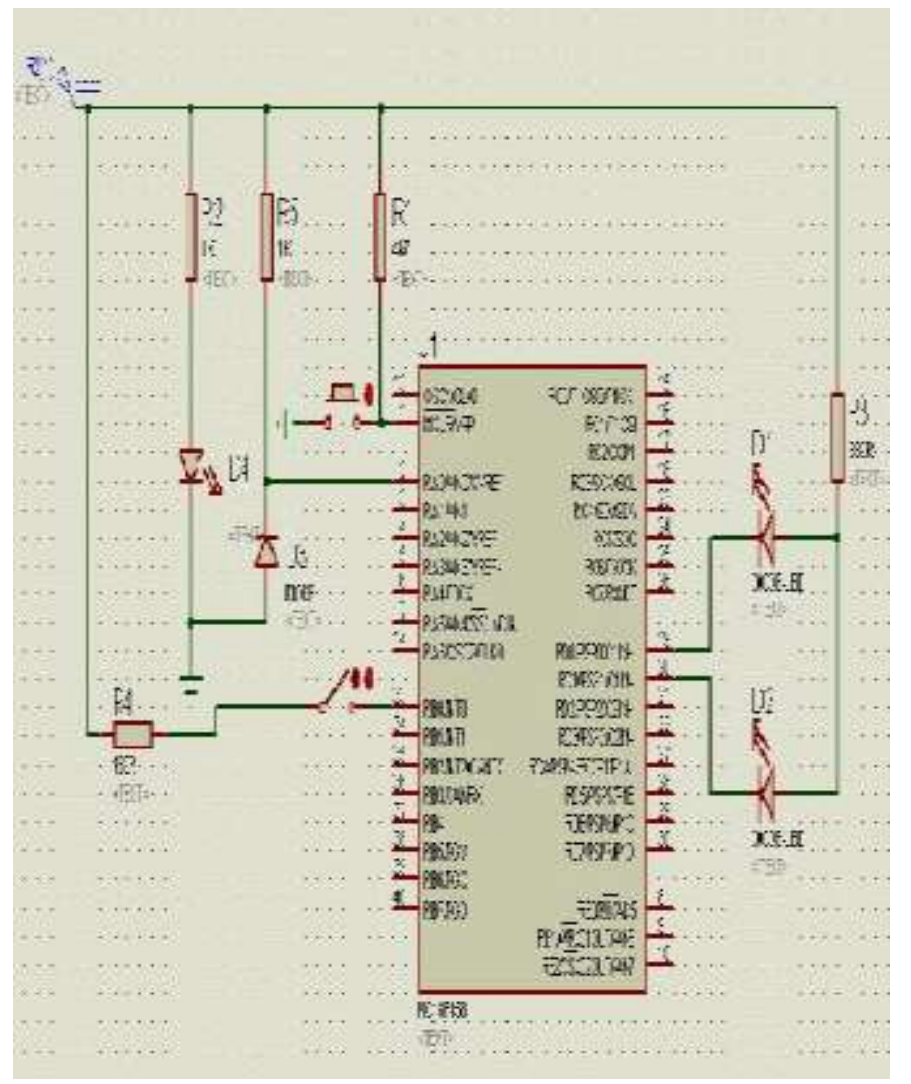

Figure4: Implementation in Proteus.

Transmitter then the voltage will decrease then it will detect rain and actuates the LED D1. If the sensor output voltage is less than $2.5 \mathrm{~V}$ then the dispersion of IR rays is more and this will actuates the LED D2 and turn off LED D1.

The above simulation in proteus shows that the program and hardware are working in coordination and the whole vision aid system for windshield assembly is running smooth. Now this concept can be implemented in real time system and can be checked for its authenticity.

\section{CONCLUSION}

All the results are tested positive and hardware's are working fine in real time environment as far as test was done. It will give a new dimension of comfort and aid to the drivers who work at night and traffic prone areas where they already have to concentrate on brakes and clutch. The removal of controlling the wipers during rain will provide them much ease and help them concentrate on the basic $\mathrm{ABC}$ (accelerator, brake and clutch) of driving.

\section{REFERENCES}

[1] The Analysis of Friction Effect in Automotive Wiper System Using Input Shaping Technique, M. A. Salim, A. Noordin, M. Z. Md. Zain, A. R. Abu Baker.

[2] Vehicular Vision System, Kenneth Scofield, Holland, MI(US); Mark L Larson, Grand Haven, MI(US); Patent No: US 7344261B2

[3] Wiper Speed Controller; Pradeep G.

[4] Development of Vision Based Control Smart Windshield Wiper System for Intelligent Vehicle; Jee-Hun Park; ManHo Kim; Hong-Jun Im; Kyung -Chang Lee; Suk Lee; Dept. of Mech. Engg., Pusan Nat. Univ.

[5] Wiper Mechanism - Hand Book of Automotive by Robert Bosch

[6] PIC 18F458 - Data sheet of PIC18F458 by Microchip.

[7] Wiper Electrical Circuits - Automotive Electrical and Electronic Systems, Second Edition by Tom Denton.

[8] History of Windshield wiper. http://www.answers.com/topic/windscreen-wiper-1.

[9] Working system of windshield wiper http://auto.howstuffworks.com/wiper.htm

[10] Rain sensor datasheet.

http://doc.diytrade.com/docdvr/365921/4271173/1189351968.pdf

[11] Wiper Motor Specification: http://www.made-inchina.com/china-

products/productviewSqsQBJYbZnVy/Wiper-Motor-BladeArm-ZD1231-2231-.html 